\title{
The susceptibility exponent of Nambu-Goto strings
}

\author{
Jan Ambjørn ${ }^{a, b}$ and Yuri Makeenko ${ }^{a, c}$ \\ ${ }^{a}$ The Niels Bohr Institute, \\ Copenhagen University, Blegdamsvej 17, \\ DK-2100 Copenhagen, Denmark \\ ${ }^{b} I M A P P$, Radboud University, \\ Heyendaalseweg 135, \\ 6525 AJ, Nijmegen, The Netherlands \\ ${ }^{c}$ Institute of Theoretical and Experimental Physics, \\ B. Cheremushkinskaya 25, \\ 117218 Moscow, Russia \\ email: ambjorn@nbi.dk makeenko@nbi.dk
}

\begin{abstract}
We compute the string susceptibility $\gamma_{\mathrm{str}}$ for the regularized Nambu-Goto string in $d$ dimensions and obtain $\gamma_{\text {str }}=1 / 2$ in $2<d<26$. This agrees with previous results obtained for lattice strings.
\end{abstract}

PACS numbers: 11.25.Pm, 11.15.Pg, 


\section{INTRODUCTION}

An important characteristics of string dynamics is the string susceptibility $\gamma_{\text {str }}$ which is determined by the pre-exponential in the entropy of surfaces with large area. For the Polyakov string of genus $g$ embedded in $d$-dimensional space-time it equals the Knizhnik-Polyakov-ZamolodchikovDavid-Distler-Kawai (KPZ-DDK) value [1]

$$
\gamma_{\mathrm{str}}^{(g)}=\gamma_{\mathrm{str}}^{(0)}+g\left(2-\gamma_{\mathrm{str}}^{(0)}\right), \quad \gamma_{\mathrm{str}}^{(0)}=\frac{c-1-\sqrt{(25-c)(1-c)}}{12}
$$

where $c=d-1 \leq 1$ is the central charge of conformal matter. Equation (1) beautifully describes a vast amount of the models with $c \leq 1$ and it follows in a simple way from the conformal invariance of the quantum Liouville action, as we will briefly review in Sec. III but it apparently breaks down for $1<c<25$ (or $2<d<26$ ), where the right-hand side of (1) becomes complex.

The non-trivial dynamical information about the coupling of geometry and matter is present already in $\gamma_{\mathrm{str}}^{(0)}$, i.e. for genus 0 , as is apparent from (1). On the other hand, $g=1$ has in this respect a special status since $\gamma_{\text {str }}^{(1)}=2$, independent of the coupling of surface geometry to matter, reflecting that the integral of the intrinsic curvature for a closed surface of genus $g=1$ is zero.

Models where $c>1(d>2)$ have been addressed for strings with an explicit UV cutoff and for the Nambu-Goto string on a hypercubic lattice it was obtained [2] (see also [3] and references therein):

$$
\gamma_{\mathrm{str}}^{(0)}=\frac{1}{2} \quad \text { for } d>2
$$

which is a typical behavior for branched polymers. The same value (2) has been obtained [4, 5] for the Nambu-Goto string with the proper-time or Pauli-Villars regularizations at $2<d<26$ in the mean-field approximation. Further, it has been argued [6] that the left equation in (1) is valid even for models with $c>1$, i.e. in particular that $\gamma_{\text {str }}^{(1)}=2$ even for these models.

The purpose of this Letter is twofold. Firstly, we repeat the computation of $\gamma_{\text {str }}^{(0)}$ of [5] for closed surfaces, such that one gets rid of the boundary effect present in [4, 5] where strings with the topology of a cylinder were considered. We do that by compactifying target space $R^{d}$ to $T^{2} \times R^{d-2}$, where $T^{2}$ is a torus of fixed periods $\beta$ and $L$. Secondly, we go beyond the mean-field approximation and show that Eq. (2) is exact for $2<d<26$. 


\section{THE SETUP FOR A TORUS}

The results [4, 5] for the long cylinder of length $L$ and circumference $\beta$ are expected to be analogous to those for the long torus modulo boundary effects which are suppressed as $\beta / L$ for the cylinder. Let us demonstrate this by explicit computations for the torus.

The setup is the following: we consider the closed bosonic string in $d$-dimensional target space, where direction 1 and 2 are compactified to a torus, $S^{1} \times S^{1}$, with periods $\beta$ and $L$. We use the Nambu-Goto action

$$
S_{N G}\left[X^{\mu}\right]=K_{0} \int \mathrm{d}^{2} \omega \sqrt{\operatorname{det} \partial_{a} X^{\mu} \partial_{b} X^{\mu}}
$$

where $K_{0}$ is the bare string tension. In addition we insist that the string winds around the targetspace torus. A classical solution, minimizing the action under the condition that it winds around the target-space torus, is then

$$
X_{\mathrm{cl}}^{1}=\beta \omega_{1}, \quad X_{\mathrm{cl}}^{2}=L \omega_{2}, \quad \rho_{a b}^{\mathrm{cl}}=\partial_{a} X_{\mathrm{cl}}^{\mu} \partial_{b} X_{\mathrm{cl}}^{\mu}=\left(\begin{array}{cc}
\beta^{2} & 0 \\
0 & L^{2}
\end{array}\right), \quad S_{N G}\left[X_{\mathrm{cl}}^{\mu}\right]=K_{0} L \beta,
$$

where the parameters $\omega_{1}, \omega_{2} \in[0,1]$ also belong to a torus with periods 1 .

In order to use the Nambu-Goto action in the path integral we follow the setup [4] for a long cylinder and introduce the Lagrange multiplier $\lambda^{a b}$ and the intrinsic metric tensor $\rho_{a b}$ such that

$$
S_{N G}\left(X^{\mu}, \lambda^{a b}, \rho_{a b}\right)=K_{0} \int \mathrm{d}^{2} \omega \sqrt{\operatorname{det} \rho_{a b}}+\frac{K_{0}}{2} \int \mathrm{d}^{2} \omega \lambda^{a b}\left(\partial_{a} X^{\mu} \partial_{b} X^{\mu}-\rho_{a b}\right) .
$$

The path integral now involves the integration over $X^{\mu}, \rho_{a b}$ and $\lambda^{a b}$. The integration over the $X^{\mu}$ is performed by writing $X^{\mu}=X_{\mathrm{cl}}^{\mu}+X_{\mathrm{q}}^{\mu}$ and performing the Gaussian integration over the quantum fluctuations $X_{\mathrm{q}}^{\mu}$. The integration over $\rho_{a b}$ requires a gauge fixing and we choose conformal gauge. We can then write

$$
\rho_{a b}(\omega)=\rho(\omega) \hat{g}_{a b}(\tau), \quad \hat{g}_{a b}(\tau)=\left(\begin{array}{cc}
1 & \tau_{1} \\
\tau_{1} & \tau_{1}^{2}+\tau_{2}^{2}
\end{array}\right), \quad \hat{g}=\operatorname{det} \hat{g}_{a b}=\tau_{2}^{2},
$$

where $\tau=\tau_{1}+i \tau_{2}$ is the modular parameter for the torus. For our choice of the classical solution (4) we have

$$
\rho_{a b}^{\mathrm{cl}}=\rho^{\mathrm{cl}} \hat{g}_{a b}\left(\tau^{\mathrm{cl}}\right), \quad \rho^{\mathrm{cl}}=\beta^{2}, \quad \tau_{1}^{\mathrm{cl}}=0, \quad \tau_{2}^{\mathrm{cl}}=\frac{L}{\beta}, \quad \sqrt{\operatorname{det} \rho_{a b}^{\mathrm{cl}}}=L \beta
$$

The integration over $X_{q}^{\mu}$ will result in a term [4, [5]

$$
(\operatorname{det} \mathcal{O})^{-d / 2}, \quad \mathcal{O}=-\frac{1}{\sqrt{\hat{g}}} \partial_{a} \lambda^{a b} \partial_{b}
$$


and similarly there is a ghost term $\operatorname{det}\left(\mathcal{O}_{\text {gh }}\right)$ from choosing the conformal gauge (6). Including the determinants in an effective action $S_{\text {eff }}\left[\lambda^{a b}, \rho\right]$ and adding a source term, we can write

$$
Z[J]=\int \frac{\mathrm{d} \tau_{1} \mathrm{~d} \tau_{2}}{\tau_{2}} \int \mathcal{D} \lambda^{a b} \int \mathcal{D} \rho \mathrm{e}^{-S_{\text {eff }}\left[\lambda^{a b}, \rho, J\right]}, \quad S_{\text {eff }}\left[\lambda^{a b}, \rho, J\right]=S_{\text {eff }}\left[\lambda^{a b}, \rho\right]+\frac{1}{2} \int \sqrt{\hat{g}} \hat{g}_{a b} J^{a b} \rho .
$$

In the mean-field approximation advocated in [4] we have that $\rho_{a b}(\omega)$ and $\lambda^{a b}(\omega)$ are independent of $\omega$ for the worldsheet coordinates we use. We can thus write

$$
\bar{\rho}_{a b}=\bar{\rho} \hat{g}_{a b}(\tau), \quad \sqrt{\operatorname{det} \bar{\rho}_{a b}}=\bar{\rho} \tau_{2}, \quad \bar{\lambda}^{a b}=\bar{\lambda} \sqrt{\hat{g}} \hat{g}^{a b}(\tau)=\bar{\lambda} \tau_{2} \hat{g}^{a b}(\tau), \quad \sqrt{\operatorname{det} \bar{\lambda}^{a b}}=\bar{\lambda}
$$

where $\bar{\rho}$ and $\bar{\lambda}$ are constants. In this case the determininants can be calculated ${ }^{1}$ and we obtain in the limit where $L \gg \beta$, using the Pauli-Villars regularization described in [5]:

$$
\begin{aligned}
& S_{\text {eff }}\left[\bar{\lambda}^{a b}, \bar{\rho}, J\right] \\
& \quad=\frac{K_{0}}{2} \bar{\lambda}^{a b}\left(\rho_{a b}^{\mathrm{cl}}-\bar{\rho}_{a b}\right)+\left[K_{0}+J-\left(\frac{d}{2 \sqrt{\operatorname{det} \bar{\lambda}^{a b}}}-1\right) \Lambda^{2}\right] \sqrt{\operatorname{det} \bar{\rho}_{a b}}-\frac{\pi(d-2)}{6} \frac{\sqrt{\operatorname{det} \bar{\lambda}^{a b}}}{\lambda^{22}} \\
& \quad=\frac{K_{0} \bar{\lambda}}{2 \tau_{2}}\left[\left(\tau_{1}^{2}+\tau_{2}^{2}\right) \beta^{2}+L^{2}\right]+\left[K_{0}(1-\bar{\lambda})-\frac{d \Lambda^{2}}{2 \bar{\lambda}}+\Lambda^{2}+J\right] \tau_{2} \bar{\rho}-\frac{\pi(d-2) \tau_{2}}{6},
\end{aligned}
$$

where $\Lambda$ is a UV cutoff.

Minimizing (11) with respect to $\bar{\rho}$, we find

$$
\bar{\lambda}(J)=\frac{1}{2}\left(1+\frac{J}{K_{0}}+\frac{\Lambda^{2}}{K_{0}}\right)+\frac{1}{2} \sqrt{\left(1+\frac{J}{K_{0}}+\frac{\Lambda^{2}}{K_{0}}\right)^{2}-\frac{2 d \Lambda^{2}}{K_{0}}}
$$

which is associated with the mean-field approximation. As was shown in [4], this minimum in $2<d<26$ is favorable to the usual classical minimum and stable under local fluctuations. At this value of $\bar{\lambda}$ the coefficient in front of $\bar{\rho}$ in Eq. 11] vanishes so this term vanishes at the minimum and we find

$$
S_{\text {eff }}[\bar{\lambda}, \bar{\rho}, J]=\frac{K_{0} \bar{\lambda}}{2 \tau_{2}}\left[\left(\tau_{1}^{2}+\tau_{2}^{2}\right) \beta^{2}+L^{2}\right]-\frac{\pi(d-2) \tau_{2}}{6}
$$

As far as the integral over the modular parameters is concerned, it has a saddle point for large $L$. The saddle point occurs at the values

$$
\bar{\tau}_{1}=0, \quad \bar{\tau}_{2}=\frac{L}{\sqrt{\beta^{2}-\frac{\pi(d-2)}{3 K_{0} \bar{\lambda}(J)}}} .
$$

For these values we have

$$
\bar{\rho}=\frac{\bar{\lambda}(J)}{\sqrt{\left(1+\frac{J}{K_{0}}+\frac{\Lambda^{2}}{K_{0}}\right)^{2}-\frac{2 d \Lambda^{2}}{K_{0}}}}\left[\beta^{2}-\frac{\pi(d-2)}{6 K_{0} \bar{\lambda}(J)}\right]
$$

\footnotetext{
${ }^{1}$ The cutoff-independent finite part of $\ln \operatorname{det}\left(-\hat{g}^{a b} \partial_{a} \partial_{b}\right)$ is given by $-\pi \tau_{2} / 3+F(\tau)$ where $F(\tau) / \tau_{2} \rightarrow 0$ for $\tau_{2} \rightarrow \infty 7$.
} 
Finally the value of the effective action in the mean-field approximation for $L \gg \beta$ reads:

$$
S_{\text {eff }}[\bar{\lambda}, \bar{\rho}, J]=K_{0} \bar{\lambda}(J) L \sqrt{\beta^{2}-\frac{\pi(d-2)}{3 K_{0} \bar{\lambda}(J)}} .
$$

Notice that for long strings with $L \gg \beta \gg 1 \sqrt{K_{0}}$ the values 14 of the modular parameters are close to the values (7) for the classical induced metric $\rho_{a b}^{\mathrm{cl}}$, and in fact $\bar{\rho}_{a b}$ becomes proportional to the classical value (7):

$$
\bar{\rho}_{a b}(J)=\frac{\bar{\lambda}(J)}{\sqrt{\left(1+\frac{J}{K_{0}}+\frac{\Lambda^{2}}{K_{0}}\right)^{2}-\frac{2 d \Lambda^{2}}{K_{0}}}} \rho_{a b}^{\mathrm{cl}}+O\left(\beta / K_{0}\right) .
$$

We finally note that for $J=0$ we can introduce renormalized target-space lengths $L_{R}, \beta_{R}$ and a renormalized coupling constant (string tension) $K_{R}$ by

$$
L_{R}=\sqrt{\frac{\bar{\lambda}(0)}{2 \bar{\lambda}(0)-1-\frac{\Lambda^{2}}{K_{0}}}} L, \quad \beta_{R}=\sqrt{\frac{\bar{\lambda}(0)}{2 \bar{\lambda}(0)-1-\frac{\Lambda^{2}}{K_{0}}}} \beta, \quad K_{R}=\left(2 \bar{\lambda}(0)-1-\frac{\Lambda^{2}}{K_{0}}\right) K_{0},
$$

such that the expressions for $S_{\text {eff }}$ and $\bar{\rho}_{a b}$ stay finite when the cutoff $\Lambda \rightarrow \infty$.

Thus we have explicitly demonstrated that the results for the long cylinder and the long torus are analogous modulo boundary effects which are suppressed for the cylinder as $\beta / L$.

\section{MEAN-FIELD APPROXIMATION FOR STRING SUSCEPTIBILITY}

The string susceptibility $\gamma_{\text {str }}$ characterizes the string entropy. In the case of Liouville gravity we have for $c<1$, after integration out the matter fields, a partition function for surfaces of genus $g:$

$$
Z(\mu)=\int \mathrm{d} m\left(\tau_{i}\right) \int \mathcal{D} \phi \mathrm{e}^{-S_{L}[\phi, \hat{g}]}, \quad S_{L}[\phi, \hat{g}]=\frac{1}{16 \pi b^{2}} \int \mathrm{d}^{2} \omega \sqrt{\hat{g}}\left(\partial_{a} \phi \partial^{a} \phi+2 b Q \hat{R} \phi+b^{2} \mu \mathrm{e}^{\phi}\right)
$$

where $b+1 / b=Q=\sqrt{(25-c) / 6}, \hat{R}$ is the scalar curvature of the metric $\hat{g}_{a b}$ and where $\mathrm{d} m\left(\tau_{i}\right)$ denotes the integration over the modular parameters $\tau_{i}$ for surfaces of genus $g$. This is the partition function for an ensemble of surfaces with cosmological constant $\mu$. The partition function $Z(A)$ of a canonical ensemble of surfaces with fixed area $A$ is related to $Z(\mu)$ by a Laplace transformation

$$
\begin{gathered}
Z(\mu)=\int_{0}^{\infty} \mathrm{d} A \mathrm{e}^{-\mu A} Z(A) \\
Z(A)=\int \mathrm{d} m\left(\tau_{i}\right) \int \mathcal{D} \phi \mathrm{e}^{-\frac{1}{16 \pi b^{2}} \int \mathrm{d}^{2} \omega \sqrt{\hat{g}}\left(\partial_{a} \phi \partial^{a} \phi+2 b Q \hat{R} \phi\right)} \delta\left(\int d^{2} \omega \sqrt{\hat{g}} \mathrm{e}^{\phi}-A\right) .
\end{gathered}
$$


By using the fact that a scaling of $A$ can be compensated by a shift $\phi \rightarrow \phi+c$ one arrives at

$$
Z(A)=A^{(g-1) Q / 2 b-1} Z(A=1):=A^{\gamma_{\mathrm{str}}^{(g)}-3} Z(A=1) .
$$

With this definition of $\gamma_{\mathrm{str}}^{(g)}-3$ one obtains (1). We remark the following: firstly, it is clear that $\gamma_{\text {str }}^{(1)}=2$ and that this scaling comes entirely from the $\delta$-function in 21). Secondly, we see that the scaling 22 formally translates into a scaling

$$
Z(\mu) \propto \mu^{2-\gamma_{\mathrm{str}}^{(g)}}, \quad \text { i.e. } \quad \frac{\mathrm{d}^{2} Z(\mu)}{\mathrm{d} \mu^{2}}=\frac{\text { const }}{\mu^{\gamma_{\text {str }}^{(g)}}}
$$

which is the reason for the notation "string susceptibility", by analogy with the susceptibilty of a spin-system, and the origin of the -3 in $\gamma_{\text {str }}-3$. Thirdly, the relation to the entropy of the number of surfaces is particularly transparent if we use the Nambu-Goto action. In this case we have formally

$$
Z(\mu)=\int \mathcal{D} S \mathrm{e}^{-\mu A(S)}=\int_{0}^{\infty} \mathrm{d} A \mathrm{e}^{-\mu A} Z(A), \quad Z(A)=\int \mathcal{D} S \delta(A(S)-A),
$$

where the integration is over embedded surfaces $S$ in the target space. In this case $Z(A)$ is just the formal number of embedded surfaces.

This number is of course infinite. In (22) this infinity has been renormalized away, but in a quantum theory where we keep the UV cutoff it will appear as an exponential growing number depending on the cutoff, but remarkably, and important for consistency of string theory, independent of the genus $g$ of the surfaces. Thus in general we will write

$$
Z(A) \propto A^{\gamma_{\mathrm{str}}^{(g)}-3} \mathrm{e}^{C(\Lambda) A}(1+O(1 / A))
$$

where $C(\Lambda)$ is a cutoff-dependent constant which can also depend on some of the other bare coupling constants of the model, and where the correction indicates that in a regularized theory we only expect the formula to be correct for areas much larger than $1 / \Lambda^{2}$ and also much larger than any dimensionful bare coupling constant in the appropriate power.

Until now we have been considering closed surfaces. Consider marking a point on the surface. For such a marked surface one would expect

$$
Z_{m}(A) \propto A^{\gamma_{\mathrm{str}}^{(g)}-2} \mathrm{e}^{C(\Lambda) A}(1+O(1 / A))
$$

simply because we can put the mark anywhere on the surface, and that should produce a factor proportional to $A$. External length-scales can be introduced either by considering vertex operators producing generalized marked points or by considering for instance strings fixed at a boundary 
in target space. Topologically, having such a boundary, say a rectangular planar loop with side lengths $L$ and $\beta$ in target space, corresponds to a surface with a marked point, and using such surfaces for the calculation of $\gamma_{\mathrm{str}}^{(g)}$ one should use formula 26 , since for $A \gg A_{\min }=L \beta$ the boundary looks essentially like a point. A similar argument applies to our present setup. Here we are not changing the topology of the surface, but we are forcing it to wind around a torus in target space which has area $A_{\min }=L \beta$, which then acts much like fixing a boundary. But in addition we should also change $g \rightarrow g-1$ in (26), since we have already, by the explicit setup, used up one of the "handles" $g$ going around the small target space torus. The large area fluctuations which contributes to the entropy will thus be fluctuations corresponding to genus $g-1$. In our case we consider surfaces of $g=1$ and by our setup, finding the large $A$ behavior of $Z(A)$, using formula 26), we will therefore determine $\gamma_{\text {str }}^{(0)}$.

Thus we consider the following fixed area partition function

$$
Z(A)=\int \frac{\mathrm{d} \tau_{1} \mathrm{~d} \tau_{2}}{\tau_{2}} \int \mathcal{D} \lambda^{a b} \int \mathcal{D} \rho \mathrm{e}^{-S_{\mathrm{eff}}\left[\lambda^{a b}, \rho\right]} \delta\left(\int \mathrm{d}^{2} \omega \rho-A\right) \stackrel{A \rightarrow \infty}{\propto} A^{\gamma_{\mathrm{str}}^{(0)}-2} \mathrm{e}^{C A} .
$$

However in order to use our mean-field results we want to get rid of the $\delta$-function in (27) and we do that by a Lagrange multiplier $J$ (i.e. in the notation of 20 we express $Z(A)$ as the inverse Laplace transform of $Z(\mu))$ :

$$
Z(A)=\int_{\uparrow} \frac{\mathrm{d} J}{2 \pi \mathrm{i}} \int \frac{\mathrm{d} \tau_{1} \mathrm{~d} \tau_{2}}{\tau_{2}} \int \mathcal{D} \lambda^{a b} \int \mathcal{D} \rho \mathrm{e}^{-S_{\mathrm{eff}}\left[\lambda^{a b}, \rho, J\right]+J A},
$$

where the integral over $J$ runs along the imaginary axis.

In the mean-field approximation we compute the integral over $J$ in (28), expanding the exponent about the saddle point at

$$
\bar{J}(A)=\sqrt{2 d \Lambda^{2} K_{0}} \frac{\left(A-A_{\min } / 2\right)}{\sqrt{A\left(A-A_{\min }\right)}}-K_{0}-\Lambda^{2}, \quad A_{\min }=L \beta .
$$

To quadratic order in $\Delta J=J-\bar{J}(A)$ we find for the exponent in 28

$$
J A-\bar{\lambda}(J) K_{0} A_{\min }=\sqrt{2 d \Lambda^{2} K_{0}} \sqrt{A\left(A-A_{\min }\right)}-\left(K_{0}+\Lambda^{2}\right) A+\sqrt{\frac{2}{d \Lambda^{2} K_{0}}} \frac{A^{3}}{A_{\min }^{2}}(\Delta J)^{2}+\ldots
$$

Integrating over $\Delta J$ along the imaginary axis and over the modular parameters $\tau_{1}$ and $\tau_{2}$ about the saddle point (14), we finally obtain

$$
\log Z(A)=-\sqrt{2 d \Lambda^{2} K_{0}} \sqrt{A\left(A-A_{\min }\right)}+\left(K_{0}+\Lambda^{2}\right) A-\frac{3}{2} \log A+\frac{1}{2} \log A_{\text {min }}+\text { const. }
$$

Comparing with the definition [27, this gives [5] $\gamma_{\mathrm{str}}^{(0)}=1 / 2$ in the mean-field approximation. 
Note also that we indeed obtain the announced form $(26)$ for $Z(A)$ :

$$
Z(A) \propto A^{-3 / 2} \mathrm{e}^{C\left(\Lambda, K_{0}\right) A}(1+O(1 / A)), \quad C\left(\Lambda, K_{0}\right)=K_{0}+\Lambda^{2}-\sqrt{2 d \Lambda^{2} K_{0}} .
$$

As discussed in [4, 5] the so-called critical point $K_{0}=K_{*}$, where scale invariance should be restored when $\Lambda \rightarrow \infty$, is precisely the point where $C\left(\Lambda, K_{0}\right)=0$,

$$
K_{*}=[(d-1)+\sqrt{d(d-2)}] \Lambda^{2},
$$

which is also the point where the constant of proportionality between $\bar{\rho}_{a b}$ and $\rho_{a b}^{\mathrm{cl}}$ in 17 diverges since $2 \lambda(0) K_{0}-\left(K_{0}+\Lambda^{2}\right)=\sqrt{\left(K_{0}+\Lambda^{2}\right)^{2}-2 d \Lambda^{2} K_{0}}$ according to $\sqrt{12}$. To obtain a finite result for $\bar{\rho}$ one has to choose $K_{0}$ infinitesimally larger than $K_{*}$, as

$$
K_{0}=K_{*}+\frac{K_{R}^{2}}{2 \Lambda^{2} \sqrt{d(d-2)}}+O\left(K_{R}^{2} / \Lambda^{6}\right),
$$

which is precisely the renormalization of $K_{0}$ given in 18 .

\section{BEYOND THE MEAN FIELD}

To account for fluctuations about the mean field, we write $\rho=\bar{\rho} \mathrm{e}^{\phi}$ and compute the effective action for $\bar{\rho}$, the slow part of the metric, by averaging over $\phi$ associated with the fast part. An important observation is that we need only the divergent part of the effective action because the finite part does not affect $\gamma_{\text {str }}$. This divergent part comes only from tadpole diagrams.

We perform the computation of (the divergent part of) the effective action (11) by pathintegrating over $\phi$. The result has the form

$$
\begin{gathered}
S_{\text {eff }}^{\operatorname{div}}\left[\lambda^{a b}, \bar{\rho}, J\right]=\int \mathrm{d}^{2} \omega \\
\left\{\frac{K_{0}}{2}\left[\lambda^{11} \beta^{2}+\lambda^{22} L^{2}-\left[\lambda^{11}+2 \lambda^{12} \tau_{1}+\lambda^{22}\left(\tau_{1}^{2}+\tau_{2}^{2}\right)\right] \bar{\rho}\right]\right. \\
\left.+\left[K_{0}+J-\left(\frac{d}{2 \sqrt{\operatorname{det} \lambda^{a b}}} f_{1}\left(b^{2}\right)-f_{2}\left(b^{2}\right)\right) \Lambda^{2}\right] \tau_{2} \bar{\rho}\right\}
\end{gathered}
$$

and involves two functions $f_{1}\left(b^{2}\right)$ and $f_{2}\left(b^{2}\right)$, coming respectively from the matter and ghost tadpoles, whose Taylor's expansions in $b^{2}$ (that multiplies the propagator of $\phi$ as usual in the Liouville field notation (see (19)) starting from 1. Since the Lagrange multiplier $\lambda^{a b}$ does not propagate, we can replace it for the torus by a constant value $\lambda^{a b}=\bar{\lambda} \sqrt{\hat{g}} \hat{g}^{a b}$ and analogously $\bar{\rho}$ is constant for our choice of the coordinates. Then the action (35) takes the form analogous to (11):

$$
S_{\mathrm{eff}}^{\mathrm{div}}[\bar{\lambda}, \bar{\rho}, J]=\frac{K_{0} \bar{\lambda}}{2 \tau_{2}}\left[\left(\tau_{1}^{2}+\tau_{2}^{2}\right) \beta^{2}+L^{2}\right]+\left[K_{0}(1-\bar{\lambda})+J-\left(\frac{d}{2 \bar{\lambda}} \Lambda_{1}^{2}-\Lambda_{2}^{2}\right)\right] \tau_{2} \bar{\rho},
$$


where for latter convenience we have introduced the notation

$$
\Lambda_{1}^{2}=f_{1}\left(b^{2}\right) \Lambda^{2}, \quad \Lambda_{2}^{2}=f_{2}\left(b^{2}\right) \Lambda^{2}
$$

The field $\bar{\rho}$ is analogous to constant fields generated in the problems of spontaneous symmetry breaking. It does not fluctuate if the volume is large and can be substituted by its value minimizing the effective action $(36)$. We finally arrive at the values of $\bar{\lambda}$ and $\bar{\rho}$

$$
\begin{aligned}
& \bar{\lambda}(J)=\frac{1}{2}\left(1+\frac{J}{K_{0}}+\frac{\Lambda_{2}^{2}}{K_{0}}\right)+\frac{1}{2} \sqrt{\left(1+\frac{J}{K_{0}}+\frac{\Lambda_{2}^{2}}{K_{0}}\right)^{2}-\frac{2 d \Lambda_{1}^{2}}{K_{0}}}, \\
& \bar{\rho}(J)=\frac{\bar{\lambda}(J)}{\sqrt{\left(1+\frac{J}{K_{0}}+\frac{\Lambda_{2}^{2}}{K_{0}}\right)^{2}-\frac{2 d \Lambda_{1}^{2}}{K_{0}}}} \beta^{2},
\end{aligned}
$$

generalizing Eqs. 12) and (15) (for $\left.\beta^{2} \gg 1 / K_{0}\right)$.

Substituting in Eq. (28), we obtain

$$
Z(A)=\int \frac{\mathrm{d} \tau_{1} \mathrm{~d} \tau_{2}}{\tau_{2}} \int_{\uparrow} \frac{\mathrm{d} J}{2 \pi \mathrm{i}} \mathrm{e}^{J A-\bar{\lambda}(J) K_{0} L \tilde{\beta} / 2}
$$

where

$$
\tilde{\beta}=\frac{\beta}{2 \tau_{2}}\left[\left(\tau_{1}^{2}+\tau_{2}^{2}\right) \frac{\beta}{L}+\frac{L}{\beta}\right] .
$$

Let us concentrate on the scaling limit when $K_{0}$ approaches the critical value

$$
K_{*}=d \Lambda_{1}^{2}-\Lambda_{2}^{2}+\Lambda_{1} \sqrt{d^{2} \Lambda_{1}^{2}-2 d \Lambda_{2}^{2}}
$$

as

$$
K_{0}=K_{*}+\frac{K_{R}^{2}}{2 \Lambda_{1} \sqrt{d^{2} \Lambda_{1}^{2}-2 d \Lambda_{2}^{2}}} .
$$

This is a generalization of the scaling limit already discussed in Eqs. (33) and (34). We then have either particle-like or string-like behavior in the scaling regime, in the terminology of [4], and

$$
\begin{aligned}
J A-S_{\mathrm{eff}}[\bar{\lambda}, \bar{\rho}, J] & =J A-K_{0} \bar{\lambda}(J) L \tilde{\beta} \\
& \stackrel{\text { s.l. }}{\rightarrow} J\left(A-\frac{L \tilde{\beta}}{2}\right)-\frac{1}{2} L \tilde{\beta} \sqrt{K_{R}^{2}+2 J\left(K_{*}+\Lambda_{2}^{2}\right)+J^{2}}-\frac{L \tilde{\beta}}{2}\left(K_{*}+\Lambda_{2}^{2}\right) .
\end{aligned}
$$

Only the domain $J \ll K_{*}$ will be essential in the integral over $J$ for $A \gg L \tilde{\beta}$ as is seen from Eq. (29), so we can drop $J^{2}$ under the square root in Eq. (43). Introducing the new variables ${ }^{2}$

$$
u=J A, \quad B=\frac{A K_{R}^{2}}{K_{*}+\Lambda_{2}^{2}}, \quad c=\frac{K_{R} L \tilde{\beta}}{2},
$$

\footnotetext{
${ }^{2}$ To avoid confusion let us note that $K_{*}+\Lambda_{2}^{2}=\sqrt{2 d \Lambda_{1}^{2} K_{*}}$.
} 
we then rewrite 43 as

$$
43=J A-\frac{L \tilde{\beta}}{2} \sqrt{K_{R}^{2}+2 J\left(K_{*}+\Lambda^{2}\right)}=u-c \sqrt{1+\frac{2 u}{B}} .
$$

These $B$ and $c$ are both large as $\sim K_{R}$ for large $K_{R}$, so the results of the previous section for the one-loop order of the expansion about the mean field can be reproduced, expanding the integral over $u$ about the saddle point

$$
\bar{u}=\frac{1}{2}\left(\frac{c^{2}}{B}-B\right)
$$

Then

$$
u-c \sqrt{1+\frac{2 u}{B}}=-\frac{B^{2}+c^{2}}{2 B}+\frac{B}{2 c^{2}}(u-\bar{u})^{2}+\ldots
$$

This results in the distribution

$$
Z(A)=\int \frac{\mathrm{d} \tau_{1} \mathrm{~d} \tau_{2}}{\tau_{2}} \mathrm{e}^{-\sqrt{2 \Lambda_{1}^{2} K_{*}} L \tilde{\beta} / 2} \frac{c}{A \sqrt{2 \pi B}} \mathrm{e}^{-B-c^{2} / 2 B},
$$

which clearly shows the same dependence on $A$ as in Eq. (31). The remaining in Eq. (48) integrals over the modular parameters do not change the $A$-dependence at large $A$ and only give the same normalization factor as in Eq. (31). How to compute these integrals will be described at the end of this section.

We can continue the analysis, introducing the new integration variable

$$
x=\sqrt{B+2 u}-\frac{c}{\sqrt{B}}
$$

to rewrite the integral over $J$ in Eq. (39) as

$$
\begin{aligned}
\int_{\uparrow} \frac{\mathrm{d} u}{2 \pi \mathrm{i} A} \mathrm{e}^{u-c \sqrt{1+\frac{2 u}{B}}} & =\mathrm{e}^{-\left(B+c^{2} / B\right) / 2} \int \frac{\mathrm{d} x}{2 \pi \mathrm{i} A}\left(x+\frac{c}{\sqrt{B}}\right) \mathrm{e}^{x^{2} / 2} \\
& =\mathrm{e}^{-\left(B+c^{2} / B\right) / 2}\left[\left.\frac{1}{2 \pi \mathrm{i} A} \mathrm{e}^{x^{2} / 2}\right|_{x_{-}} ^{x_{+}}+\left.\frac{c}{2 \mathrm{i} A \sqrt{2 \pi B}} \operatorname{Erfi}(x / \sqrt{2})\right|_{x_{-}} ^{x_{+}}\right.
\end{aligned}
$$

with

$$
x_{ \pm}=\sqrt{B \pm 2 \mathrm{i} \infty}-\frac{c}{\sqrt{B}} .
$$

This gives precisely the distribution (48) whose one-loop approximation calculated by the expansion (47) is thus exact.

The remaining integrals over the modular parameters $\tau_{1}$ and $\tau_{2}$ in Eq. 48 can be easily computed at large $L \beta$ by the saddle point given by Eq. 14 and which is justified by large $L \beta \sqrt{2 d \Lambda_{1}^{2} K_{*}}$. Accounting for the fluctuations about this saddle point, the final result for the long torus is

$$
\log Z(A)=\frac{L \beta}{2} \sqrt{2 d \Lambda_{1}^{2} K_{*}}-\frac{3}{2} \log A+\frac{1}{2} \log A_{\min }+\text { const. }, \quad A_{\min }=L \beta
$$


which reproduces the scaling regime of (31) for $\Lambda_{1}=\Lambda$. We have thus demonstrated that this behavior is exact, yielding $\gamma_{\text {str }}^{(0)}=1 / 2$ for bosonic string in $2<d<26$ to all orders.

\section{CONCLUSION}

The main result of this paper is that $\gamma_{\mathrm{str}}^{(0)}=1 / 2$ for Nambu-Goto bosonic strings in target-space dimensions $2<d<26$. Our setup was designed to avoid any problem with tachyonic modes of the bosonic string. The origin of this half-integer value is the square-root dependence of the effective action on $J$ through $\bar{\lambda}(J)$ given by Eq. 12 which is the true minimum. The usual classical string ground state is stable only for $d<2$, where zero-point fluctuations indeed increase the effective action. In the formal limit $d<2$ we thus expect the standard results from the Liouville theory, and it is seen explicitly from the formulas that the limit $d \rightarrow 2^{+}$is somewhat singular.

The value $\gamma_{\mathrm{str}}^{(0}=1 / 2$ is clearly outside the standard range of KPZ-DDK. We used explicitly the Pauli-Villars regularization when calculating the effective action. However, it agrees with the value obtained for bosonic strings in $2<d<26$ using a hyper-cubic lattice [2]. It is interesting to understand if our effective bosonic string theory, which has $\gamma_{\mathrm{str}}^{(0)}=1 / 2$, can still be viewed as a conformal invariant worldsheet theory.

\section{Acknowledgment}

Y.M. was supported by the Russian Science Foundation (Grant No.20-12-00195). Y.M. thanks the Theoretical Particle Physics and Cosmology group at NBI for the long-term warm hospitality.

[1] V.G. Knizhnik, A.M. Polyakov and A.B. Zamolodchikov, Fractal structure of $2 D$ quantum gravity, Mod. Phys. Lett. A 3, 819 (1988).

F. David, Conformal field theories coupled to $2 D$ Gravity in the conformal gauge, Mod. Phys. Lett. A 3, 1651 (1988).

J. Distler and H. Kawai, Conformal field theory and 2D quantum gravity, Nucl. Phys. B 321, 509 (1989).

[2] B. Durhuus, J. Frohlich and T. Jonsson, Selfavoiding and planar random surfaces on the lattice, Nucl. Phys. B 225, 185 (1983); Critical behavior in a model of planar random surfaces, Nucl. Phys. B 240, 453 (1984), Phys. Lett. B 137, 93 (1984).

[3] J. Ambjorn, B. Durhuus and T. Jonsson, Quantum geometry. A statistical field theory approach, Cambridge (UK) Univ. Press (1997). 
[4] J. Ambjorn and Y. Makeenko, String theory as a Lilliputian world, Phys. Lett. B 756, 142 (2016) arXiv:1601.00540; Scaling behavior of regularized bosonic strings, Phys. Rev. D 93, 066007 (2016) arXiv:1510.03390; Stability of the nonperturbative bosonic string vacuum, Phys. Lett. B 770, 352 (2017) arXiv:1703.05382 [hep-th]].

[5] J. Ambjorn and Y. Makeenko, The use of Pauli-Villars' regularization in string theory, Int. J. Mod. Phys. A 32, 1750187 (2017) arXiv:1709.00995 [hep-th]].

[6] B. Durhuus, Multispin systems on a randomly triangulated surface, Nucl. Phys. B 426, 203 (1994) [arXiv:9402052 [hep-th]].

[7] J. Polchinski, Evaluation of the one loop string path integral, Commun. Math. Phys. 104, 37 (1986).

[8] A. B. Zamolodchikov, On the entropy of random surfaces, Phys. Lett. 117B, 87 (1982).

S. Chaudhuri, H. Kawai and S. Tye, Path integral formulation of closed strings, Phys. Rev. D 36, 1148 (1987).

I. K. Kostov and A. Krzywicki, On the entropy of random surfaces with arbitrary genus, Phys. Lett. B 187, 149 (1987). 